\title{
STABILITY AND PERFORMANCE OF FEEDBACK CONTROL SYSTEMS WITH TIME DELAYS
}

\author{
M.S. Ali†, Z.K. Hou and M.N. Noori \\ Mechanical Engineering Department, Worcester Polytechnic Institute, Worcester, MA 01609, U.S.A
}

\begin{abstract}
This paper investigates the time delay effects on the stability and performance of active feedback control systems for engineering structures. A computer algorithm is developed for stability analysis of a SDOF system with unequal delay time pair in the velocity and displacement feedback loops. It is found that there may exist multiple stable regions in the plane of the time delay pair, which contain time delays greater than the maximum allowable values obtained by previous studies. The size, shape and location of these stable and unstable regions depend on the system parameters and the feedback control gains. For systems with multiple stable regions, the boundaries between the stable and unstable regions in the plane of the time delay pair are explicitly obtained. The delay time pairs that forms these boundaries are called the critical delay time pairs at which the steady-state response becomes unbounded. The conclusions are valid for both large and small delay times. For any system with multiple stable regions, preliminary guidelines obtained from an explicit formula are given to find the desirable delay time pair(s). When used, these desirable delay time pair(s) not only stabilize an unstable system with inherent time delays, but also significantly reduce the system response and control force. For any system with multiple stable regions, these desirable delay time pair(s) are above the maximum allowable delay times obtained by previous studies. Numerical results, for both steady-state and transient analysis, are given to investigate the performance of delayed feedback control systems subjected to both harmonic and real earthquake ground motion excitations. 1997 Published by Elsevier Science Ltd
\end{abstract}

Key words--desirable time delay pairs, feedback control system, multiple stable regions, multiple unstable regions, stability, time delay pairs

\section{INTRODUCTION}

In real active control systems, time delays in control action are caused by acquisition of response and excitation data, on-line data processing and computation of control force, and application of control forces. Efforts have been devoted to minimize the time delays. However, time delay cannot be eliminated totally due to its inherent nature, even with today's advanced technology.

Effects of time delay on the stability and performance of control systems has drawn attention of many investigators in different engineering disciplines, including structural systems [1-8], chemical processes [9-15], remotely controlled undersea and aerospace robots and structures [16], and manufacture processes $[17,18]$. In general, time delay in active control systems causes unsynchronized application of the control forces, and this unsynchronization not only degrades the system performance, but also causes instability of the system response.

Recently, in the area of active structural control, discussions are mainly given to the delayed feedback control systems with either delay time in one feedback loop or equal delay time in both the velocity and the displacement feedback loops. The delay time is considered to be small relative to the

tTo whom correspondence should be addressed. natural period of the system and, therefore, perturbation technique can apply. Abdel-Rohman [2] considered the effect of small time delay on the stability of a distributed-parameter structure with the velocity feedback by using Taylor's series expansion and neglecting the second order terms. Mcgreevy et al. [5] and Chung et al. [19] performed experimental studies on a SDOF system with equal delay times in both velocity and displacement feedback loops. Chung et al. [8] also conducted experimental studies on an MDOF system with equal delay times. Hou and Iwan [4] conducted a study on the effect of time delay on actively controlled SDOF model with equal delay times and subjected to harmonic excitation. A closed-form solution was given for the critical time delay families at which the response of the system becomes unbounded. The results are valid for both large and small delay times. However, as a preliminary study, only the steadystate response was discussed. Pu and Kelly [7] used the frequency response analysis for an SDOF system with equal delay times to find the maximum allowable time delay beyond which the system becomes unstable. In their analysis both steadystate and transient behaviors were considered. Agrawal et al. [20] performed a stability analysis for an SDOF model with equal delay times and found a closed form solution for the critical delay time or the maximum allowable time delay. 
This paper addresses effects of time delays on stability and performance of a SDOF system with unequal time delays in the relative velocity and displacement feedback loops. It is found that multiple stable regions may exist in the plane of the time delay pairs, which contains time delay pairs higher than the maximum allowable time delay obtained from previous studies $[7,20]$. Stable and unstable regions and the boundaries that separate them were identified by using a computer algorithm which utilizes Newton-Raphson method. Preliminary guidelines, obtained from an explicit formulas, are given to find the desirable delay times and a new control strategy is proposed by using these desirable delay times. The discussion is given for a general case of two unequal time lags in the relative velocity and displacement feedback loops. The results may reduce to those for special cases where the time delay exists in only one of the feedback loops, or time delays in these two feedback loops are equal.

\section{FORMULATION}

A non-dimensional form of the governing equation of motion for a SDOF feedback control system with a time delay $u_{1}$ in the relative velocity feedback loop and a time delay $u_{2}$ in the relative displacement feedback loop can be written as follows:

$$
\begin{aligned}
\frac{\mathrm{d}^{2}}{\mathrm{~d}^{2}} x(\bar{t}) & +2 \varsigma \frac{\mathrm{d}}{\mathrm{d} \bar{t}} x(\bar{t})+x(\bar{t}) \\
& +K_{2} \frac{\mathrm{d}}{\mathrm{d} t} x\left(\bar{t}-u_{1}\right)+K_{1} x\left(\bar{t}-u_{2}\right)=F(\bar{t})
\end{aligned}
$$

where

$$
\begin{gathered}
\vec{t}=\omega_{n} t, u_{1}=\omega_{n} \tau_{1}, u_{2}=\omega_{n} \tau_{2}, \\
K_{2}=2 \zeta \beta^{*} / c, K_{1}=\gamma^{*} / k,
\end{gathered}
$$

in which $\bar{t}$ is the non-dimensional time, $u_{1}$ and $u_{2}$ are the non-dimensional time lags, $\omega_{n}$ and $\zeta$ are the natural frequency and damping ratio of the system, respectively. $K_{2}$ and $K_{1}$ are the non-dimensional feedback control gains. $\beta^{*}$ and $\gamma^{*}$ are the control gains. $c$ and $k$ are the viscous damping constant and the stiffness of the system. $F(\bar{t})$ is the nondimensional external excitation. Zero initial conditions are assumed in this study.

Laplace transform of Equation (1) leads to a transfer function expressed as

$$
\frac{X(s)}{F(s)}=\frac{1}{s^{2}+2 \zeta s+1+2 \zeta \beta s \mathrm{e}^{-u_{1} s}+\gamma \mathrm{e}^{-u_{2} s}}=\frac{1}{Q(s)},
$$

where $s=\sigma+j \delta$ and $\delta=\omega / \omega_{n}$.

For any feedback control system with a given delay time pairs, the stability of the control system can be determined by finding the roots of the characteristic equation $Q(s)$. If at least one root of $Q(s)$ has a positive real part, i.e. $\sigma>0$, the system becomes unstable. All values of the time delay pairs associated with unstable control systems construct unstable regions in the plane of the time delay pair. A computer algorithm is developed which uses the Newton-Raphson's method to solve for the roots of the characteristic equation. For special cases where the Newton-Raphson's method cannot find root(s) with a positive real part, Nyquist method is included in the computer algorithm to double check the stability of the system. Only for systems with multiple stable regions, can the boundaries between the stable and unstable regions in the plane of the time delay pair be explicitly obtained. These boundaries are constructed from all the time delay pairs at there associated frequency ratios which satisfy the equation $Q(j \delta)=0$. This means that all the roots of the characteristic equation have zero real part, i.e. $\sigma=0$.

Define the critical delay pair as a pair of time lags, i.e. $u_{1}$ and $u_{2}$, satisfying the equation $Q(j \delta)=0$. It can be shown that a necessary and sufficient condition for existence of the critical delay pair for a linear feedback control system with nonzero damping and control gains is given by:

$$
|p(\delta)| \leq 1
$$

where $p(\delta)$, referred as the characteristic function of the feedback control system (1), is defined as:

$$
p(\delta)=\frac{1}{4 \zeta \gamma \beta}\left[\delta^{3}+\delta\left(4 \zeta^{2}\left(1-\beta^{2}\right)-2\right)+\frac{1}{\delta}\left(1-\gamma^{2}\right)\right] \text {. }
$$

The definition of the characteristic function should be modified for undamped systems and/or zero control gain(s). Equation (4) is useful in determining the possible frequency ratios that may obtain an infinite sets of critical delay time pairs which satisfies $Q(j \delta)=0$.

For any frequency expressed in terms of the frequency ratio $\delta=\omega / \omega_{n}$, the corresponding critical delay pairs can be found as:

$$
u_{1}=\frac{1}{\delta}(\bar{A}+\bar{B}), u_{2}=\frac{1}{\delta}(\bar{A}+\bar{B}),
$$

where $\bar{A}$ and $\bar{B}$ are given by

$$
\begin{aligned}
\bar{A}= & \tan ^{-1}\left(\frac{2 \zeta \delta}{\delta^{2}-1}\right)+\tan ^{-1}\left(\frac{K_{2} \delta \cos \bar{B}+K_{1} \sin \bar{B}}{K_{2} \delta \sin \bar{B}+K_{1} \cos \bar{B}}\right) \\
& +2 k_{2} \pi, \bar{B}=\frac{1}{2} \sin ^{-1}(P(\delta))+k_{1} \pi
\end{aligned}
$$

in which $k_{1}$ and $k_{2}$ are two arbitrary integers which are chosen such that both $u_{1}$ and $u_{2}$ are non-negative. It is clear that there exists an infinite set of the critical delay pairs for any particular frequency ratio and they are periodically distributed. For the 


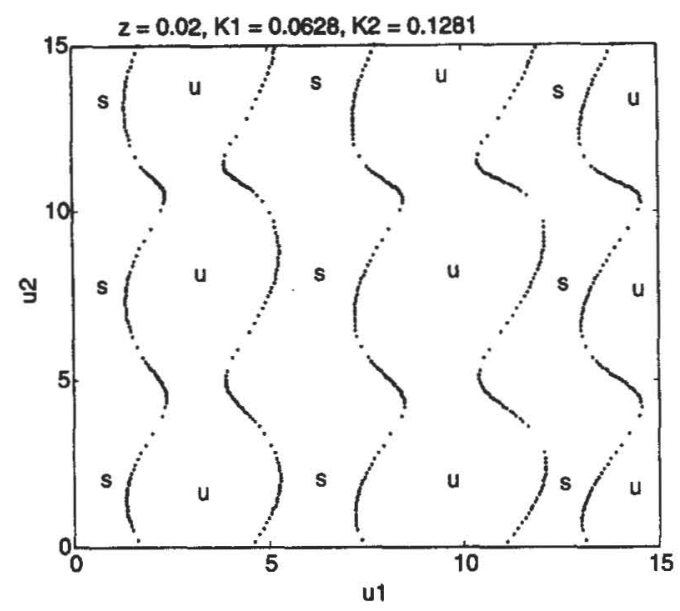

Fig. 1. $u_{1}-u_{2}$ plane.

special case of equal time delay the results reduce to those in Iwan and Hou [3].

\section{Numerical examples}

In this section, numerical results are presented for a linear SDOF feedback control system with $2 \%$ damping ratio. The feedback control gains are $K_{1}=0.0628$ and $K_{2}=0.1281$. The system's maximum allowable time delay pair is $u_{1}=u_{2}=1.3358$, as determined from the methodology in Agrawal et al. [20].

Figure 1 shows the critical time delay pairs, stable and unstable regions for this system in the plane of the time delay pair. The stable regions are marked with the letter "s", while the unstable regions are marked with the letter " $u$ ".

Any point in the unstable region represents a delay pair which causes an unstable control. It is observed that there are multiple regions of stability for this system. The critical delay pairs in these regions may be greater than the maximum allowable time delay pair as determined from the pre-

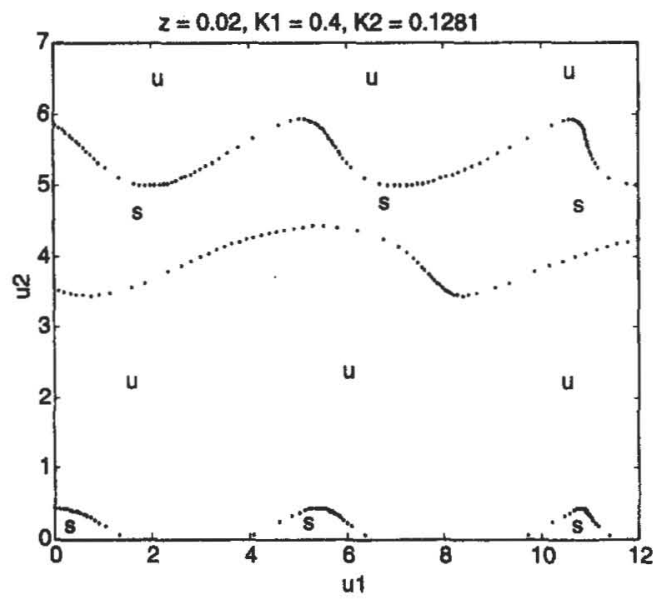

Fig. 3. $u_{1}-u_{2}$ plane.

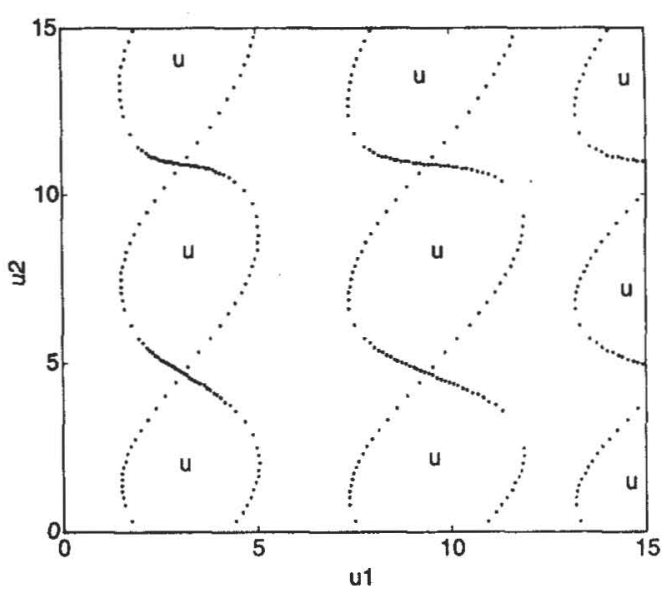

Fig. 2. $u_{1}-u_{2}$ plane

vious approaches. As observed, the total area of the stable region(s) shrinks as $u_{1}$ increases and these regions would shrink more if lower damping ratio were used. It is found that for sufficiently large natural frequency of the system, the unstable regions disappear and the system becomes unconditionally stable no matter how much the time delay values are in the feedback loops. The above results can be justified by Equations (4) and (5).

When increasing the damping ratio, while keeping the feedback gains fixed, the total area of the stable region(s) increases. Figure 2 below shows the case when the damping ratio increases to 0.0327 such that $P(\delta)=-1$, in Equation (5), has two repeated solutions for the frequency ratio.

As the damping ratio keeps increasing, the unstable region(s) keep shrinking. The unstable region(s) disappears when $P(\delta)=1$, in Equation (5), has two repeated solutions for the frequency ratio.

Now let us consider the effect of changing the feedback gain $K_{1}$, while keeping $K_{2}$ and the damping ratio fixed. Figure 3 below shows the case when

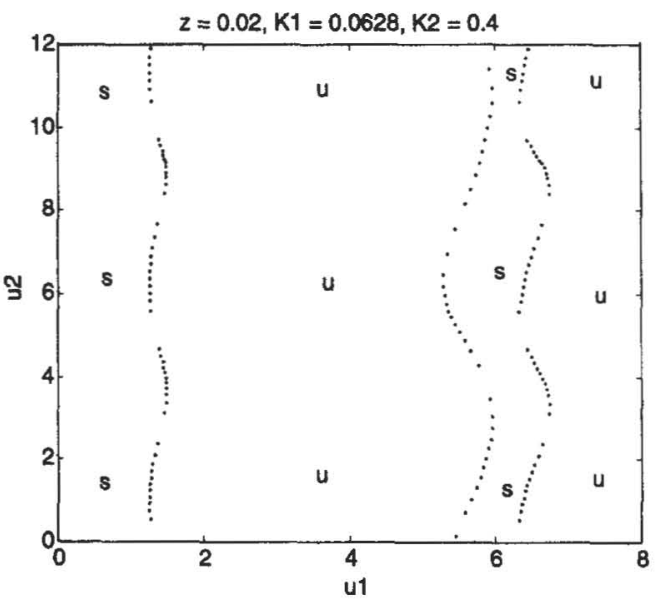

Fig. 4. $u_{1}-u_{2}$ plane. 


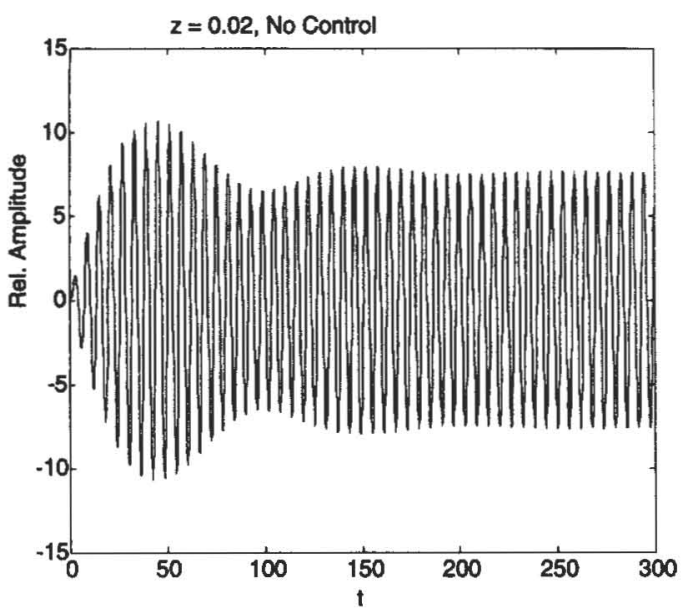

Fig. 5. Non-dimensional transient response with no control.

$K_{1}=0.4$. It is clear from this figure that increasing $K_{1}$ results in decreasing the total area of the stable regions.

Now let us consider the effect of changing the feedback gain $K_{2}$, while keeping $K_{1}$ and the damping ratio fixed. Figure 4 below shows the case when $K_{2}=0.4$. It is clear from this figure that increasing $K_{2}$ results also in decreasing the total area of the stable regions. However, it is clear that the boundaries and the shape of the stable and unstable regions, in Fig. 3, are different from Fig. 4.

\section{Desirable delay time pairs}

In this paper, the desirable delay time pairs, for any system, are defined as the delay time pairs that are located in the stable regions of the $u_{1}-u_{2}$ plane, and in the same time they should give us the minimum steady-state response and the minimum steady-state control force.

The steady-state response of any vibration system can be presented in non-dimensional form. This form is called the steady-state magnification factor $(D)$. The steady-state magnification factor $(D)$ for the system in Equation (1) can be written as:

$$
D=\frac{1}{\sqrt{A^{2}+B^{2}}}
$$

where

$$
\begin{gathered}
A=1-\delta^{2}+K_{1} \cos \left(\delta u_{2}\right)+K_{2} \delta \sin \left(\delta u_{1}\right) \\
B=2 \zeta \delta-K_{1} \sin \left(\delta u_{2}\right)+K_{2} \delta \cos \left(\delta u_{1}\right) .
\end{gathered}
$$

Equation (8) is general and can be used for systems with feedback control and no delays (i.e. $u_{1}=u_{2}=0$ ) and for systems with no feedback control (i.e. $K_{1}=K_{2}=0$ ).

In order to find the delay time pairs which are going to give us the minimum steady-state magnification factors, we need to take the first derivative

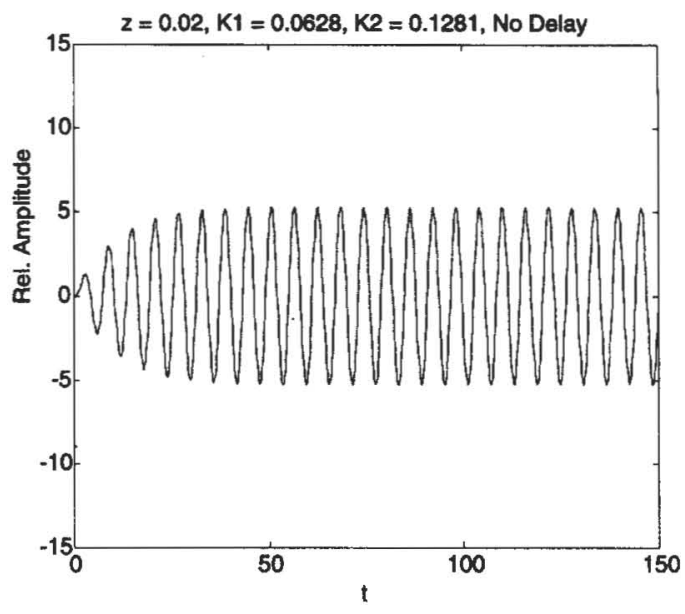

Fig. 6. Non-dimensional transient response with control and no delay.

of Equation (8) with respect to $u_{1}$ and $u_{2}$, respectively, and then equate the two equations to zero and solve them. The following analytical expressions result:

$$
\begin{aligned}
& u_{1}=(1 / \delta)\left\{\arctan \left[\left(1-\delta_{2}\right) /(2 \zeta \delta)\right]+2 k_{1} \pi\right\}, \\
& u_{2}=(1 / \delta)\left\{\arctan \left[(-2 \zeta \delta) /\left(1-\delta^{2}\right)\right]+2 k_{2} \pi\right\},
\end{aligned}
$$

in which $k_{1}$ and $k_{2}$ are two arbitrary integers which are chosen such that both $u_{1}$ and $u_{2}$ are nonnegative. For any given $\delta, k_{1}$, and $k_{2}$, there is a single delay time pair that can be obtained from Equation (9). It is clear that the delay time pairs obtained in Equation (9) depend on both the damping ratio of the system and the frequency ratio and do not depend on the feedback gains.

For any given system, the desirable delay time pairs are extracted from the delay time pairs which are evaluated from Equation (9) and they should be located in the stable regions of that particular system. Once the desirable delay time pairs and their corresponding frequency ratios are identified, one can calculate the desirable minimum steady-state magnification factors by using Equation (8).

For systems with equal delay time pair (i.e. $u_{1}=u_{2}=u$ ), the analytical expression for the delay time pairs which are going to give us the minimum steady-state magnification factors is as follows:

$u=(1 / \delta)\left\{\arctan \left[\left(\alpha K_{2} \delta-\lambda K_{1}\right) /\left(\alpha K_{1}+\lambda K_{2} \delta\right)\right]+2 k \pi\right\}$,

where $\alpha=1-\delta^{2}, \lambda=2 \zeta \delta$, and $k$ is an arbitrary integer which is chosen such that $u$ is non-negative.

From this equation, it is clear that these time delay pairs depend on the damping ratio of the system, frequency ratio and the feedback gains. 


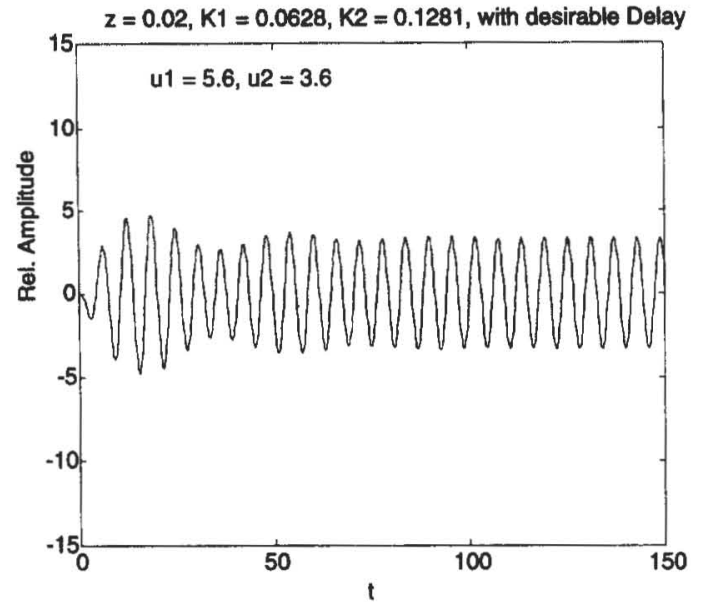

Fig. 7. Non-dimensional transient reponse wigh control and desirable delay.

\section{Effectiveness of using the desirable delay time pairs}

In order to investigate the effectiveness of using the desirable delay time pairs, let us define $X R$ as the ratio of the steady-state magnification factor of a system with delayed feedback control to the steady-state magnification factor of the undelayed feedback control system:

$$
X R=\sqrt{\frac{\left(1-\delta^{2}+K_{1}\right)^{2}+\delta\left(2 \zeta+K_{2}\right)^{2}}{A^{2}+B^{2}}} .
$$

It can be shown that $X R$ represents also the feedback control force ratio.

For any given system, the ratio $X R$ in Equation (10) would give us a good picture of how effective the use of a desirable delay time pair in the feedback loops is in reducing both the dynamic response and the control force when compared to a non-delayed feedback loops for the same system.

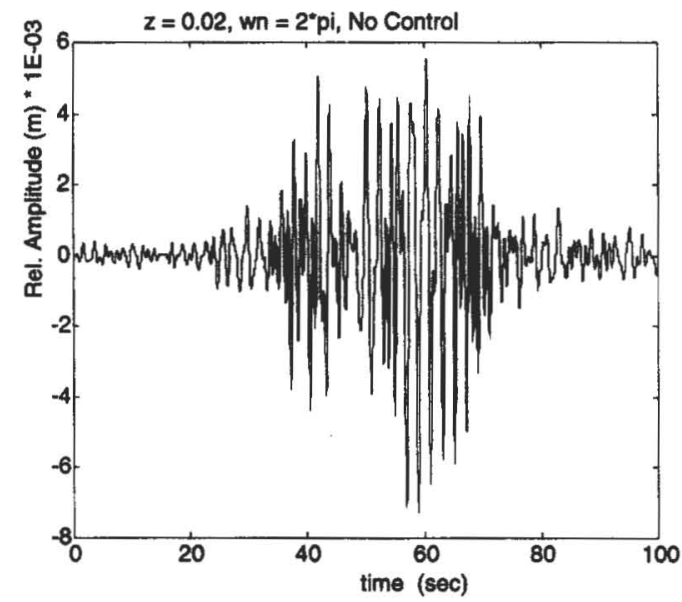

Fig. 9. Transient response with no control.

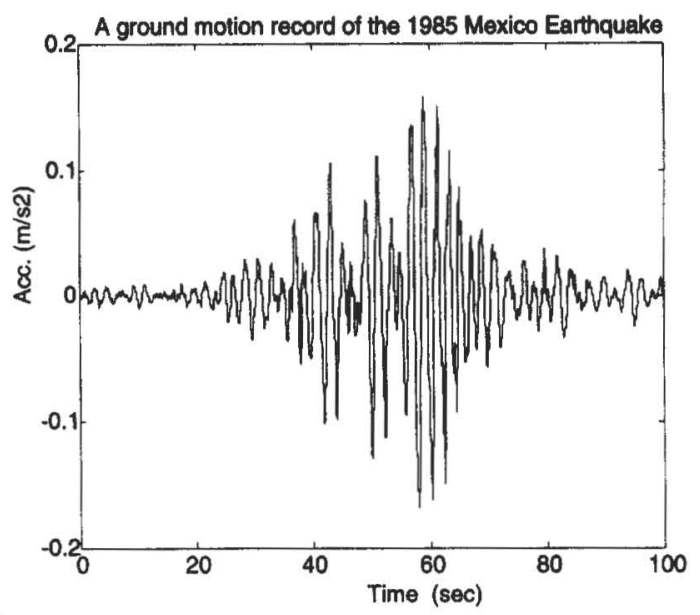

Fig. 8. 1985 Mexico Earthquake Record.

Figure 1 suggests a new control strategy for time delay compensation techniques. Due to the existence of the multiple stable regions, one might choose a particular delay pair, referred as the desirable delay pair, such that the system is stable and an acceptable suppression of vibration may be achieved. The approach is specially attractive for control system with large inherent time delay. Instead of minimizing the inherent time delay in the control system, which is usually difficult and costly, this approach provides a new feasible and efficient approach. The idea was tested for the system in Fig. 1 and some results are included in following figures.

Numerical results are provided for the dynamic response of the above-mentioned feedback control system subjected to harmonic excitations and real earthquake ground motion records from both 1985 Mexico and $1940 \mathrm{El}$ Centro Earthquakes. Zero initial conditions are assumed in all the calculations. For the purpose of comparison, three cases are con-

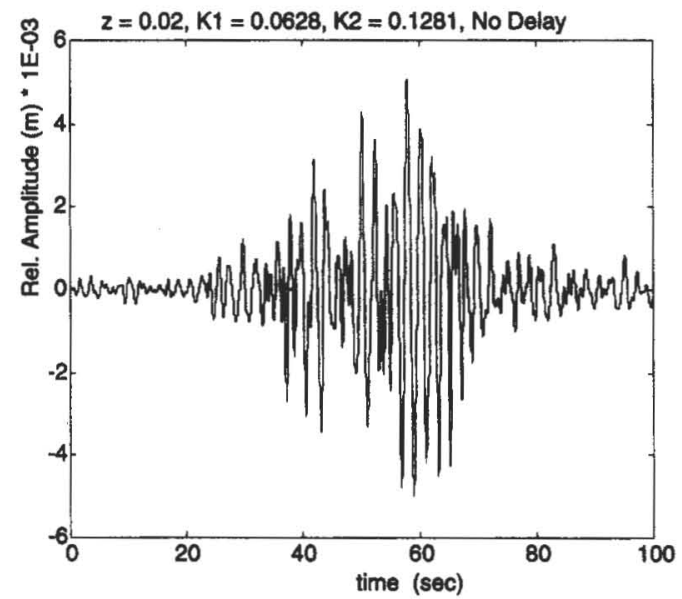

Fig. 10. Transient response with control and no delay. 


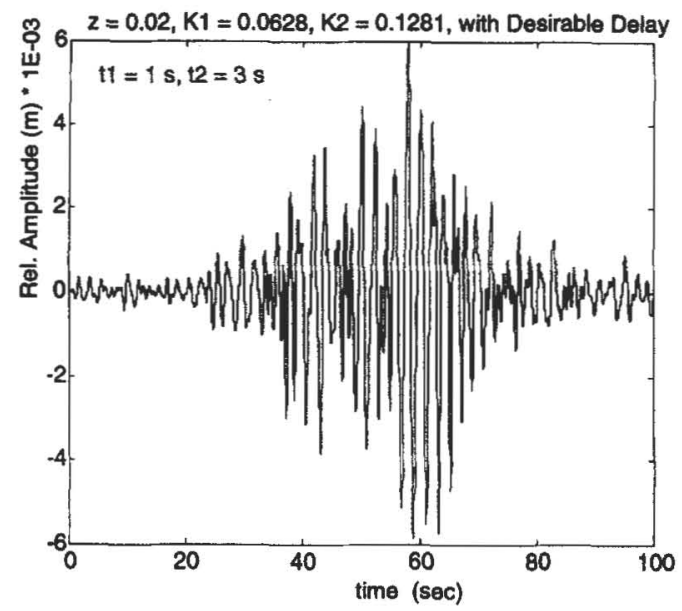

Fig. 11. Transient response with control and desirable delay.

sidered for each excitation: (1) the transient response of the system with no control; (2) the transient response of the system with control and no delay in the feedback loops; and (3) the transient response of the system with desirable delay time pair in the feedback loops.

Figure 5 presents the non-dimensional transient response of the system with no control subjected to a resonance harmonic excitation. For sufficiently large time, the system response achieves its steadystate. Figure 6 presents results for the non-dimensional transient response of the feedback control system with control and no delay.

Significant reduction in amplitude is achieved as high as $34 \%$. Figure 7 illustrates the response for the same feedback control system but with a desirable delay time pair, $u_{1}=5.6$ and $u_{2}=3.6$, in the feedback loops. A higher reduction in amplitude is achieved as high as $56.8 \%$.

The comparison is repeated for the same system subjected to a 1985 Mexico Earthquake ground

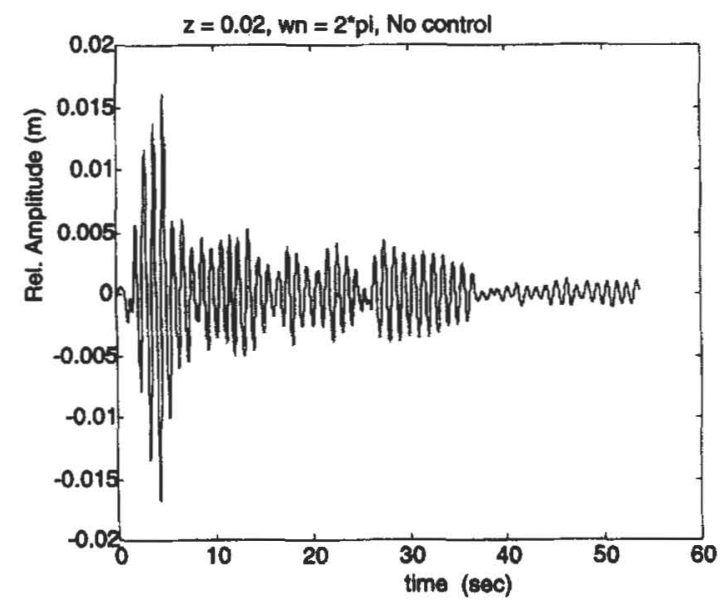

Fig. 13. Transient response with no control.

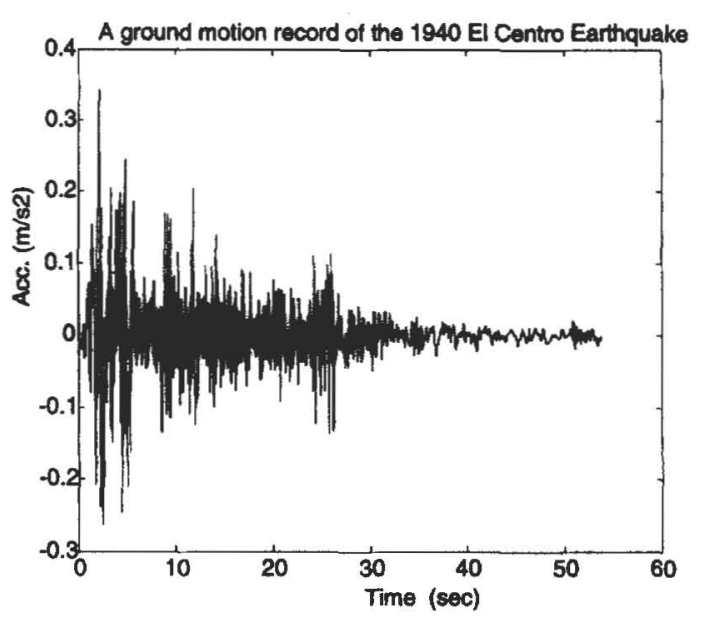

Fig. 12, 1940 El Centro earthquake record.

motion record as shown in Fig. 8, which is a narrow-band excitation with long duration. Figure 9 presents the transient response of a system with $1 \mathrm{~Hz}$ natural frequency and $2 \%$ damping ratio with no control.

Figure 10 presents the results for the transient response of the feedback control system with control and no delay. Significant reduction in amplitude is achieved as high as $23.55 \%$. Figure 11 illustrates the response for the same feedback control system, but with a desirable delay time pair, $t_{1}=1 \mathrm{~s}$ and $t_{2}=3 \mathrm{~s}$, in the feedback loops. An acceptable reduction of $12.14 \%$ in amplitude is achieved.

Similar comparison is performed for the same system to $1940 \mathrm{El}$ Centro Earthquake record which is shown in Fig. 12. Figure 13 presents the transient response of a system with $1 \mathrm{~Hz}$ natural frequency and $2 \%$ damping ratio with no control. Figure 14 presents the results for the transient response of the feedback control system with control and no delay. Significant reduction in amplitude is achieved as

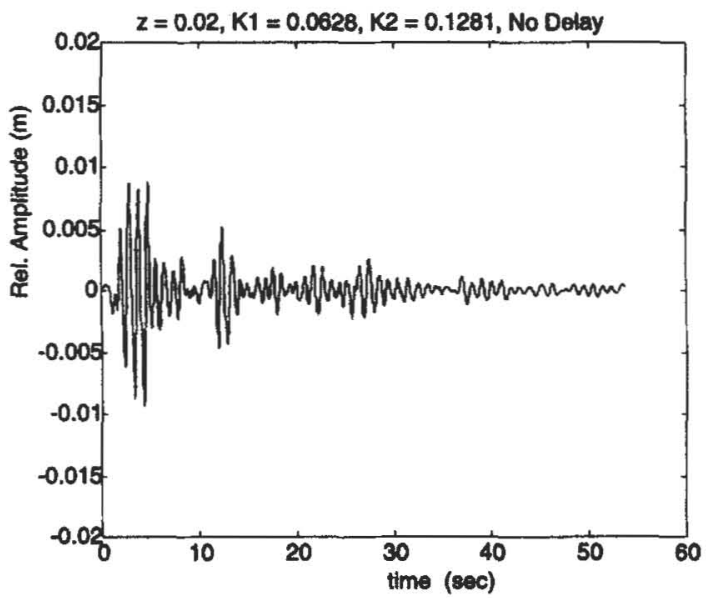

Fig. 14. Transient response with control and no delay. 


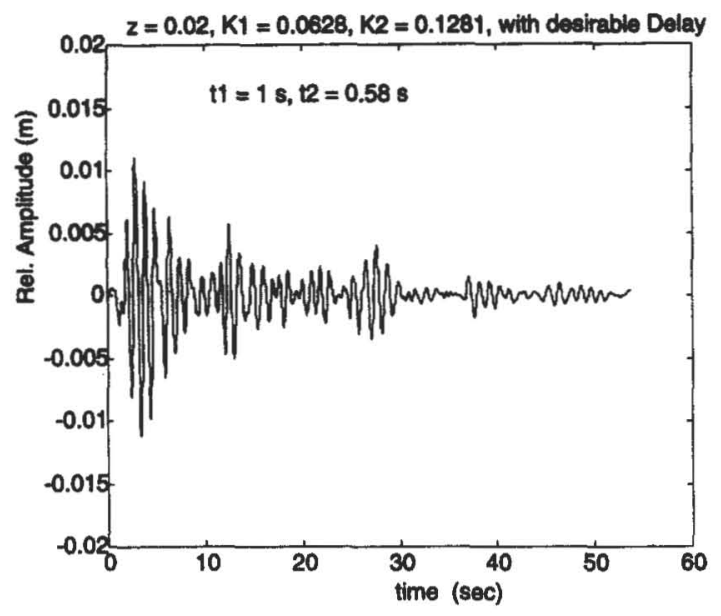

Fig. 15. Transient response with control and desirable delay.

high as $44 \%$. Figure 15 illustrates the response for the same feedback control system, but with a desirable delay time pair, $t_{1}=1 \mathrm{~s}$ and $t_{2}=0.58 \mathrm{~s}$, in the feedback loops. An acceptable reduction of $30.62 \%$ in amplitude is achieved.

\section{CONCLUSIONS}

Stability analysis of an SDOF system with unequal time delay pair in the velocity and in the displacement feedback loops has been presented. A computer algorithm is developed which uses Newton-Raphson's method complemented with the Nyquist method to investigate the stability of any given system. It is shown that there may exist multiple stability regions for the feedback control system in the plane of the time delay pair, $u_{1}$ and $u_{2}$. For systems with multiple stable regions, analytical expressions are found for the critical delay pairs which help in identifying the boundaries between stable and unstable regions and the results are valid for both large and small values of the time delay pair. These regions contains critical time delay pair(s) greater than the maximum allowable time delay obtained by previous investigators for systems with equal time delay pairs in the velocity and the displacement feedback loops. It is found that the size, shape, location and distribution of the stable regions depend on the system parameters as well as the values of the feedback gains for the velocity and displacement loops. It is found that, by increasing the damping ratio, while keeping the feedback gains fixed, the total area of the stable region(s) increases. On the other hand, it is found that, by increasing one of the feedback gains while keeping the damping ratio and the other feedback gain fixed, the total area of the stable region(s) shrinks. The results were verified by the dynamic response of a linear SDOF feedback system subjected to harmonic excitation and real earthquake ground motion data. It CAS 66/2-3-C has been shown that for the given system, system response may become unbounded if the critical delay pair is used. The system response can be stabilized if a desirable delay pair is chosen from the stable regions, that is even higher than the maximum allowable delay time. Preliminary guidelines are given to find these desirable delay time pairs from explicit formulas. A significant reduction in the transient displacement response of the controlled systems with certain desirable time delay pairs can be obtained. The approach of using the desirable delay pairs might be more economical and practical for control systems with large inherent time delay in the feedback control loop which cannot be totally eliminated even by using sophisticated and very expensive equipment.

\section{REFERENCES}

1. Abdel-Rohman, M. Structural control considering time delay effect. Transactions of the CSME, 1985, 9, 224-227.

2. Abdel-Rohman, M. Time-delay effects on actively damped structures ASCE. Journal of Engineering Mechanics, 1987, 113, 1709-1719.

3. Iwan, W. D. and Hou, Z. K., Some aspects of the use of active control algorithms in vibration suppression. In Proceedings of the U.S. National Workshop on Structural Control Research, ed. G. W. Housner and S. F. Masri, October 1991.

4. Hou, Z. K. and Iwan, W. D., Reliability problem of active control algorithms caused by time delay. In Proceedings of the 10th World Conference on Earthquake Engineering, Vol. 4. Balkema, Rotterdam, 1992 , pp. 2149-2153.

5. Mcgreevy, S., Soong, T. T. and Reinhorn, A. M., An experimental study of time delay compensation in active structural control. In Proceedings of the SEM 6th International Modal Analysis Conference, Orlando, 1988, pp. 733-739.

6. T. T. Soong, Active Structural Control: Theory and Practice, Wiley, New York, 1990.

7. Pu, J.-P. and Kelly, J.M. Active control and seismic isolation. Journal of Engineering Mechanics ASCE, 1991, 117, 2221-2236.

8. Chung, L.L., Lin, R.C., Soong, T.T. and Reinhorn, A.M. Experimental study of active control for MDOF seismic structures. Journal of Engineering Mechanics, ASCE, 1989, 115, 1609-1627.

9. Hammarstrom, L.G. and Gros, K.S. Adaptation of optimal control theory to systems with time delays. International Journal of Control, 1980, 32, 329-357.

10. Inamdar, S.R., Kumar, V. and Kulkarni, B.D. Dynamics of reacting system in the presence of time delay. Chemical Engineering Science, 1991, 46, 901-908.

11. Mutharasan, R. and Luus, R. Analysis of time delay system by series approximations. AIchE Journal, 1975, 21, 567-571.

12. Oh, S.H. and Luus, R. Optimal feedback control of time-delay systems. AIchE Journal, 1976, 22, 140-147.

13. Ross, D.W. Controller design for time lag systems via a quadratic criterion. IEEE Transactions on Automatic Control, 1971, 16, 664-672.

14. Schell, M. and Ross, J. Effect of time delay in rate processes. Journal of Chemistry and Physics, 1986, 85, 6489-6503. 
15. Soliman, M.A. and Ray, W.H. Optimal feedback control for linear-quadratic systems having time delays. International Journal of Control, 1972, 15, 609-627.

16. Van de Vegte, J.M.E., Milgram, P. and Kwong, R.H. Teleoperator control model: effects of time delay and imperfect system knowledge. IEEE Transactions on Systems, Man and Cybernetics, 1990, 20, 12581272.

17. Rubin, $O$. Delay lines in analog computer studies part I and II. IEEE Transactions on Industrial Electronics and Control Instrumentation, 1964, IECI-11, 36-42.
18. Smith, O.J.M. Closer control of loops with dead time. Chemical Engineering Progress, 1957, 53, 217-219.

19. Chung, L.L., Reinhorn, A.M. and Soong, T.T. Experiments on active control of seismic structures'. ASCE, Journal of Engineering Mechanics, 1988, 114, 241-255.

20. Agrawal, A.K., Fujino, Y. and Bhartia, B. Instability due to time delay and its compensation in active control of structures. Earthquake Engineering and Structural Dynamics, 1993, 22, 211-224. 